\title{
First Principal Treatment of Size of Inflaton Potential, Inflaton Mass, Scale Factors, and Frequency of Emitted Radiation, Using Linde and Padmanabhan Models of Early Cosmology and BEC
}

\author{
Andrew Beckwith \\ Physics Department, College of Physics, Chongqing University, Chongqing, China \\ Email: rwill9955b@gmai.com
}

How to cite this paper: Beckwith, A. (2021) First Principal Treatment of Size of Inflaton Potential, Inflaton Mass, Scale Factors, and Frequency of Emitted Radiation, Using Linde and Padmanabhan Models of Early Cosmology and BEC. Journal of High Energy Physics, Gravitation and Cosmology, 7, 1477-1483. https://doi.org/10.4236/jhepgc.2021.74090

Received: September 17, 2021

Accepted: October 22, 2021

Published: October 25, 2021

Copyright $\odot 2021$ by author(s) and Scientific Research Publishing Inc. This work is licensed under the Creative Commons Attribution International License (CC BY 4.0).

http://creativecommons.org/licenses/by/4.0/

\begin{abstract}
Using a Linde reference, as well as another one from Padmanabhan for calculation of how the early universe expands, we obtain, by default the coefficient of scale factor expansion, $t$ to the alpha value, with alpha being approximately the square root of five in value. We from there make an estimate as to the number of initial particles produced in the very beginning, which leads us to conclude that a graviton, would be a preferred initial by product. The argument as to gravitons, also reflects a choice of how the decay of initial BEC condensates of Planck sized black holes would commence, using the work produced by Chavanis, as to BEC condensates and black holes. The object will be to obtain initial frequency spread plus strength of GW production plus a suggestion as to what polarization state may be accessible from initial conditions.
\end{abstract}

\section{Keywords}

Scalar-Tensor Gravity, BEC, Scale Factor, Massive Gravitons

\section{Introduction}

We use the work by Linde, [1] as to what is given on page 257 which translates into a different model of the Hubble expansion

$$
H^{2}=\frac{8 \pi}{3 M_{p}^{2}} \cdot\left(\frac{\dot{\phi}^{2}}{2}+V(\phi)\right)-\frac{H^{2}}{M^{2}} \cdot\left(H^{2}+2 \frac{\ddot{H}}{H}-\left(\frac{\dot{H}}{H}\right)^{2}\right)
$$


Here, we are assuming, a fourth order substitution of a particle of mass $M$ much less than Planck mass being produced, with say, Planck mass being approximately the size of the initial black holes, and with micro black holes being produced by

$$
\begin{aligned}
& M^{2}=\tilde{\alpha}^{2} M_{p}^{2} \\
& \tilde{\alpha} \ll 1
\end{aligned}
$$

We also will be using a scale factor for the expansion of the universe which is [2]

$$
\begin{aligned}
& a \approx a_{\min } t^{\gamma} \\
& \Leftrightarrow \phi \approx \sqrt{\frac{\gamma}{4 \pi G}} \cdot \ln \left\{\sqrt{\frac{8 \pi G V_{0}}{\gamma \cdot(3 \gamma-1)}} \cdot t\right\} \\
& \Leftrightarrow V \approx V_{0} \cdot \exp \left\{-\sqrt{\frac{16 \pi G}{\gamma}} \cdot \phi(t)\right\}
\end{aligned}
$$

Equation (1) applied with this information will lead to the following if we apply the following Planck unit normalizations, of Planck mass, Planck time, and $G$ to one, then

$$
\begin{aligned}
& M^{2}=\tilde{\alpha}^{2} M_{p}^{2} \rightarrow \tilde{\alpha}^{2} \\
& \tilde{\alpha} \ll 1 \\
& t \equiv \beta t_{P} \rightarrow \beta \\
& \beta \geq 1 \\
& G \rightarrow 1
\end{aligned}
$$

Applying Equation (1), to Equation (4) will then lead to the following

$$
2 \tilde{\alpha}^{2} \beta^{2}=v^{2}-3
$$

So

$$
\begin{aligned}
& \tilde{\alpha}^{2} \beta^{2} \approx o(1) \\
& \Rightarrow v \approx \sqrt{5}
\end{aligned}
$$

This is for a time bigger than Planck time, and subsequent particles less than Plank mass presumably being produced by BEC black holes, of which we will be referencing [3]

$$
\begin{aligned}
& m \approx \frac{M_{P}}{\sqrt{N_{\text {gravitions }}}} \cdot M_{P} \\
& M_{B H} \approx \sqrt{N_{\text {gravitions }}} \cdot l_{P} \\
& R_{B H} \approx \sqrt{N_{\text {gravitions }}} \\
& S_{B H} \approx k_{B} \cdot N_{\text {gravitions }} \\
& T_{B H} \approx \frac{T_{P}}{\sqrt{N_{\text {gravitions }}}}
\end{aligned}
$$

What we will be doing if the Gravitons travel at nearly the speed of light is to make the Identification of a spatial displacement of graviton BEC condensate 
BHs having a spatial dimension of the following value

$$
R_{B H} \approx \beta l_{P}
$$

We will elaborate upon the implications of this in the third chapter of this paper after we go to asking if we next construct a minimum value of the inflaton mass which we will try relating to formation of early universe Planck mass black holes.

\section{Examining the Role of Our Model on the Formation of an Inflaton Mass}

In order to do this, we will be looking at what is given in [4] namely

$$
\ddot{\phi}+3 H \dot{\phi}+m_{\phi}^{2} \phi=0
$$

Then we have the following, namely

$$
m_{\phi}^{2} \approx\left(\frac{1-3 v}{\beta^{2} t_{P}^{2}}\right) /\left(\log \left(\sqrt{\frac{8 \pi G V_{0} \beta^{2} t_{P}^{2}}{v(3 v-1)}}\right)\right)
$$

In order to have this inflation mass be $>0$, and not imaginary, we would need to have

$$
V_{0}<\frac{v \cdot(3 v-1)}{8 \pi G \beta^{2} t_{P}^{2}} \underset{G, t_{P} \rightarrow 1}{\longrightarrow} \frac{v \cdot(3 v-1)}{8 \pi \beta^{2}}
$$

So then after algebra, we would have that the inflaton mass, so defined would lead to the inflaton if it broke up leading to Planck sized black holes, according to [5], i.e. if we had that Equation (10) would have masses greater than Planck mass, according to then [5] we would have

Quote

We show that fragmentation of the inflaton into long-lived spatially localized oscillon configurations can lead to copious production of black holes.

End of quote

Note in reference 5 the term oscillon is described as a generalization of the inflaton.

i.e. the formation of a black hole, or numerous black holes, aside from the breakup of the inflaton, would also require a minimum size of the inflaton, which would then necessitate it being above one Planck mass. The mechanism of the generation of black hole generation is implied to be at the end of inflation in [5] as given by the following quote from [5].

Quote

In figure 2 we display the cosmological history of the setup, showing energy density evolution of the inflaton, oscillons, $\mathrm{PBHs}$ as well as radiation from reheating. During inflation, the inflaton dominates the Universe.

End of quote 
To wit, this means of course that there would be a time for generation of black holes greater than a simple rendition of Planck time as the ending point which is consistent with the model we so far have been using. And then we can go after assuming a net production of black holes being broken up by density according to this procedure mass $\mathrm{m}$ breaks up for $\mathrm{m}$ as given by [6]

$$
m \approx \frac{8 \pi R(\text { radius })^{3} \cdot \rho}{3}
$$

for our application and, we obtain for black holes a breakup criterion for mass $m$.

Black holes if

$$
m \approx-\left(\frac{4 \pi \rho}{3}\right) \cdot\left(1+3 \cdot \frac{\rho_{G}}{p_{G}}\right) \cdot \frac{8 m^{3}}{M_{P}^{6}}
$$

So we can have the start of breakup of black holes right after the end of inflation, and this is in tandem with looking at i.e. we have then that the black holes, which maybe produced by the inflaton are broken up when the mass of a black hole, call it $\mathrm{m}$, is leading to a generalized density of the universe as given by

$$
\rho_{B H} \approx M_{P}^{4} \cdot\left(\frac{M_{P}}{m}\right)^{2} \cdot \frac{3}{32 \pi} \cdot \frac{1}{\left|1+3 \cdot\left(w_{Q}=\frac{P_{Q}}{\rho_{Q}}\right)\right|}
$$

Here we have that

$$
\frac{P_{Q}}{\rho_{Q}}=\frac{\frac{\dot{\phi}^{2}}{2}-V(\phi)}{\frac{\dot{\phi}^{2}}{2}+V(\phi)} \approx \frac{3 v^{2}-2 v}{-3 v^{2}}
$$

Given what we have so far computed, with, say $v \approx \sqrt{5}$, or maybe a bit larger, we may have for sufficiently large time value, a situation where we cannot just assume for the sake of modeling, that Equation (15) would be -1 which is what is usually assumed, i.e. that the density is the negative value of the pressure. i.e. a more nuanced picture forms, and that we would be looking at, with analysis, a situation for which we have would be for finite $v$ a situation not exactly close to the phenomenology right at the start of inflation for which we would have the time $t$, for the production of gravitons, i.e. we would not have Equation (15) equal to -1 which would only occur if we have $v$ becoming almost infinite in value.

\section{Results about What This Procedure Implies for the Frequency of Emitted Gravitons}

We have that the time, $t$ is proportional to the following, so if gravitons travel at nearly the speed of light

$$
\begin{aligned}
& t \approx \beta t_{P} \underset{t_{P} \rightarrow 1}{\longrightarrow} \beta \\
& \Rightarrow \beta \approx \sqrt{n} \equiv \sqrt{N_{g}}
\end{aligned}
$$


If so, following Equation (7) we will have that in the regime of space-time for which

$$
\begin{aligned}
& \text { If so } \\
& \begin{array}{l}
E \approx \frac{k_{B} T}{2} \approx \hbar \omega \\
T \approx T_{P} / \sqrt{N_{g}} \\
\Rightarrow \omega \approx \frac{1.855 \times 10^{43} \mathrm{~Hz}}{\beta \approx \sqrt{n} \equiv \sqrt{N_{g}}}
\end{array}
\end{aligned}
$$

This would be the frequency, as to initial graviton production, assuming that one is using Equation (7) above for BEC style condensate treatment of gravitons, with, say for a certain regime of space-time we have that we are assuming a real inflaton mass, which is larger than Planck mass, which puts a surprisingly strong damper on the value of $V_{0}$ according to Equation (7), indicating an e fold value of about 60. Or about a reduction of $10^{-27}$ in terms of the frequency Equation (17) would permit in terms of measurement platforms for GW within our solar system. The interesting result is that this value for frequency would DECREASE, unless I am totally wrong, for situations where per unit value of space, we have graviton production increasing in the situation for which primordial black holes, break up, that Equation (17) plays an unexpected role of scaling which I, as author did not expect. In addition, [7] gives cautionary background which must be considered carefully.

\section{Conclusion, What Does All This Sort of Formulation Say as to the Number of Physical Polarized States of GW Radiation?}

From [8] we have that if there exist scalar-tensor models of gravity, as an extension of what is in GW production, that we have more than two polarization states, as to gravity. We assert that in this article, that this supposition is correct. In doing so, we will review arguments as to the chance we have scalar-tensor models of cosmology involved and what this says about our measurement problem. Reference [9] as given will refer to scalar-tensor gravity, and we note what is near their conclusion

Quote, from reference [9]

In the post Planck era gravity should be treated classically, while the matter fields still behave quantum mechanically. 'Quantum Field Theory in Curved Space-Time' (QFT in CST), treats gravity classically while the energy-momentum tensor is quantized. For General Theory of Relativity, it is expressed as $\mathrm{G} \mu \nu=\langle\mathrm{Tb} \mu \nu>$, where the quantum operator $<\mathrm{Tb} \mu \nu>$ is a suitably renormalized expectation value. The perturbative semiclassical approximation under consideration in this subsection, is quite different from QFT in CST. In this technique, the perturbed parts of the metric and matter fields are treated as quantum mechanical operators, keeping the background parts classical [10] [11] [12]. Since, we derive the perturbation spectra generated 
from the quantum fluctuations in an early scalar field dominated accelerating (inflation) phase, therefore this approach appears to be more legitimate

End of quote

We argue that we are doing a modification of GR, but one which still treats Gravity semi classically if one looks at Equation (1) as from Linde [1]. But that the Quantum mechanical nature of assuming Bose-Einstein, condensates, as given in Equation (7) will be a gateway to scalar-tensor gravity.

Further work, as given in [9] having their Equation (3.31), Equation (3.32), Equation (3.33) and Equation (3.34) are ways of imposing quantum conditions upon the scalar fields, as cited in [9]. We will state unequivocally that a similar process is obtained by linking imports and the phenomenonology of Equation (2) and Equation (7).

Note that in our approach, we are making the following assumption, namely, that.

Following Conformal Equivalence theory, multidimensional theories of gravity are conformal equivalent to theories of usual General Relativity in 4 dimensions with an additional scalar field.

Reference [13] also has a way of linkage of linking scalar-tensor theories with the methodology of DE, which in our approach may be relevant, especially if there is any later phenomenological evidence that massive gravitons, are indeed an active candidate as to DE. i.e., the idea of a release of a sea of gravitons, in future work, due to the breakup of black holes, as specified by Equation (14) and Equation (15) may be pertinent to the development of DE. As cited in [14] the idea of massive gravitons may relate to $\mathrm{DE}$ and the first step toward falsifiable data sets may lie in obtaining rigorous limits as to the polarization states, allowed, which will be the subject of a future inquiry. And we wish to state that [15] as to GW from small relic black holes must be taken into consideration, as well as the physics which is in reference [16].

\section{Dedication of This Paper, and Acknowledgements}

Prof. Padmanabhan passed away on 17 September 2021. I thereby dedicate this paper to him in lieu as to what I have learned through his texts and research articles. This work is indeed partially founded on an approach of Prof. Padmanabhan, and I dedicate what I did in this document to his memory.

\section{Conflicts of Interest}

The author declares no conflicts of interest regarding the publication of this paper.

\section{References}

[1] Andrei, L. (1990) Particle Physics and Inflationary Cosmology. CRC Books, New York.

[2] Thanu, P. (2006) An Invitation to Astrophysics. World Press Scientific, World Scientific Series in Astronomy and Astrophysics: Volume 8, Singapore. 
[3] Chavanis, P.H. (2012) Self-Gravitating Bose-Einstein Condensates. In: Calmet, X., Ed., Quantum Aspects of Black Holes. Fundamental Theories of Physics, Springer, Cham, 151-194. https://doi.org/10.1007/978-3-319-10852-0_6

[4] Martin, B. and Tilman, P. (2019) Yet Another Introduction to Dark Matter, the Particle Physics Approach. Springer Nature, Heidelberg.

[5] Cotner, E., Kusenko, A. and Takhistov, V. (2018) Primordial Black Holes from Inflaton Fragmentation into Oscillons. Physical Review D, 98, Article ID: 083513. https://arxiv.org/abs/1801.03321 https://doi.org/10.1103/PhysRevD.98.083513

[6] Freeze, K., Brown, M.G. and Kinney, W. (2012) The Phantom Bounce: A New Proposal for an Oscillatory Cosmology. In: Mersini-Houghton, L. and Vaas, R., Eds., The Arrows of Time, A Debate in Cosmology, Springer, Berlin, Heidelberg, 149-156. https://doi.org/10.1007/978-3-642-23259-6_7

[7] Grøn, Ø. (2018) Predictions of Spectral Parameters by Several Inflationary Universe Models in Light of the Planck Results. Universe, 4, 15.

https://doi.org/10.3390/universe4020015

[8] Li, F.Y., Wen, H., Fang, Z.Y., et al. (2020) Electromagnetic Response to High-Frequency Gravitational Waves Having Additional Polarization States: Distinguishing and Probing Tensor-Mode, Vector-Mode and Scalar-Mode Gravitons. The European Physical Journal C, 80, Article No. 879. https://doi.org/10.1140/epjc/s10052-020-08429-2

[9] Mandal, R., Sarkar, C. and Sanyal, A.K. (2018) Early Universe with Modified Scalar-Tensor Theory of Gravity. Journal of High Energy Physics, 2018, Article No. 78. https://arxiv.org/abs/1801.04056 https://doi.org/10.1007/JHEP05(2018)078

[10] Hwang, J.-C. (1997) Quantum Fluctuations of Cosmological Perturbations in Generalized Gravity, Class. Quantum Gravity, 14, 3327. https://doi.org/10.1088/0264-9381/14/12/016

[11] Hwang, J.-C. (1996) Unified Analysis of Cosmological Perturbations in Generalized Gravity. Physical Review D, 53, 762. https://doi.org/10.1103/PhysRevD.53.762

[12] Hwang, J.-C. and Noh, H. (1996) Cosmological Perturbations in Generalized Gravity Theories. Physical Review D, 54, 1460. https://doi.org/10.1103/PhysRevD.54.1460

[13] Paolo, C. and Filippo, V. (2017) Dark Energy after GW170817 and GRB170817A. Physical Review Letters, 119, Article ID: 251302. https://doi.org/10.1103/PhysRevLett.119.251302

[14] Drakova, D. and Doyen, G. (2019) Localized Gravitons as Essence of Dark Energy: Implications for Accelerated Cosmic Expansion. Journal of Physics: Conference Series, 1275, Article ID: 012055. https://doi.org/10.1088/1742-6596/1275/1/012055

[15] Dolgov, A. and Ejlli, D. (2011) Relic Gravitational Waves from Light Primordial Black Holes. Physical Review D, 84, Article ID: 024028.

https://arxiv.org/abs/1105.2303 https://doi.org/10.1103/PhysRevD.84.024028

[16] Utpal, S. (2008) Particle and Astro Particle Physics. Taylor and Francis, Boca Raton, Florida. 